\title{
La depuración de las matronas de Madrid tras la Guerra Civil
}

\author{
Dolores Ruiz-Berdún $\left({ }^{*}\right)$ y Alberto Gomis (**) \\ $\left.{ }^{*}\right) \quad$ Unidad Docente de Matronas de Madrid, E.U.E. Cruz Roja de Madrid. \\ ruizberdun@gmail.com \\ (**) Universidad de Alcalá. \\ alberto.gomis@uah.es
}

Dynamis

[0211-9536] 2012; $32(2): 439-465$
Fecha de recepción: 17 de junio de 2011

Fecha de aceptación: 26 de enero de 2012

SUMARIO: 1.-Introducción. 2.-La implicación de varios Ministerios en la depuración de las matronas de Madrid. 3.-Depuración y sanción de las trabajadoras en la Universidad Central. 4.-Depuración y sanción de las matronas que actuaron como funcionarias de prisiones. 5.- La depuración de las matronas por el Colegio de Médicos de Madrid. 6.-Matronas sancionadas por el Colegio de Médicos de Madrid. 7.-Conclusiones.

RESUMEN: Las matronas de Madrid habían conseguido incorporarse a diferentes puestos de la Administración en los años previos a la Guerra Civil. Esto explica que, tras la contienda, la depuración de las mismas se llevara a cabo por parte de diferentes instancias. En el presente trabajo se analizan los procesos de depuración de las que trabajaban en diferentes departamentos de la Universidad Central, las que habían obtenido plaza de funcionarias de prisiones y las depuradas por el Colegio de Médicos de Madrid, que constituyeron la gran mayoría. Después de explicar cómo se formaban los expedientes de depuración por parte del juez instructor correspondiente y de cómo se resolvieron, nos detenemos en los casos de las matronas que recibieron las sanciones más elevadas y en los motivos aducidos por los respectivos jueces para proponerlas.

PALABRAS CLAVE: matronas, depuración, Universidad Central, funcionarias de prisiones, Colegio de Médicos de Madrid.

KEY WORDS: midwives, purge, University of Madrid, female prison officers, College of Medicine of Madrid. 


\section{Introducción}

El primer título universitario al que pudo optar la mujer en España fue el de matrona, titulación exclusivamente femenina hasta bastante después de la Guerra Civil. No obstante, su incorporación al funcionariado público en Madrid fue muy tardía, debido a que en la mayoría de las ocasiones, las matronas habían quedado excluidas de los puestos oficiales desde los que se realizaba la atención al parto. Estos cargos habían sido ocupados tradicionalmente por otro tipo de profesionales sanitarios varones que no deseaban perder sus prerrogativas, como los cirujanos-comadrones primero y los tocólogos titulares después ${ }^{1}$.

Desde mediados del siglo XIX hasta prácticamente los años de la Segunda República, el número de matronas que se titulaban en Madrid cada año fue significativamente menor si lo comparamos con otros puntos de examen, como Barcelona ${ }^{2}$. Pese a ello, en los años previos a la Guerra Civil ya era posible encontrar matronas, en diferentes organismos públicos madrileños, en empleos para los que contar con la titulación era un requisito, o al menos un mérito que mejoraba sus posibilidades. Hay que hacer notar la doble titulación que tenían muchas de ellas, pues en numerosas ocasiones habían completado también los estudios de practicanta y en algunos, aunque muchos menos, los de maestra. Estos estudios, además de situarlas con un nivel cultural superior al de la media de mujeres de la época, les facilitaban el acceso al mundo laboral. Por lo general, estas matronas siguieron perteneciendo al Colegio Oficial de Matronas de Madrid, como prueba la lista de colegiadas que elabora el Colegio Oficial de Matronas de Madrid en 1935 y que obra en nuestro poder ${ }^{3}$. En la misma figuran 375 matronas ordenadas alfabéticamente, siendo la numeración más alta que corresponde a una de ellas, el número 487.

Antes de finalizar la guerra, con la radicalización que sufrieron ambos bandos incursos en la contienda, se iniciaron una serie de medidas represoras sobre diferentes colectivos. Por un lado, el gobierno republicano

\footnotetext{
1. Precisamente, el estudio del desarrollo histórico de la profesión de matrona en Madrid hasta la Guerra Civil, constituye el tema nuclear de la tesis doctoral que hemos ultimado y cuya defensa se ha llevado a cabo durante el proceso de edición de este volumen.

2. Ortiz Gómez, Teresa. Las matronas y la transmisión de saberes científicos sobre el parto. Arenal [artículo en internet]. 1999; 6 (1): 55-79. Disponible en: http://hdl.handle.net/10481/15378 [citada 1 Jun 2011].

3. Lista de matronas colegiadas. Colegio Oficial de Matronas de Madrid, 1935.
} 
aprobó por decreto de 21 de julio de 1936 (Gaceta de Madrid, 22 de julio de 1936) la cesantía en sus puestos «de todos los empleados que hubieran tenido participación en el movimiento subversivo, o fuesen notoriamente enemigos del Régimen». Por la otra parte, el bando franquista dispuso la depuración con la máxima rapidez del personal civil que dependía del Estado, para lo cual promulgó la Ley de 10 de febrero de 1939, fijando normas para la depuración de los funcionarios públicos (BOE, 14 de febrero de 1939). Según la misma, cada uno de los Ministerios Civiles que constituían la Administración de Estado procedería a la investigación de la conducta social, religiosa y fundamentalmente política seguida por los funcionarios públicos. La depuración constaba de dos fases: una informativa y otra resolutiva. La resolución de los expedientes correspondía al Ministerio respectivo. El personal diplomático y el docente se depuró con arreglo a unas normas especiales ${ }^{4}$.

La depuración del personal sanitario (médicos de asistencia pública domiciliaria, farmacéuticos, matronas y practicantes) se atuvo a la Ley de 10 de febrero. A pesar de que su nombramiento hubiera correspondido a las corporaciones locales, no se les aplicó la Orden de 12 de marzo de 1939 sobre depuración de funcionarios de la Administración Local, en relación con el Movimiento Nacional (BOE, 14 de marzo de 1939), ya que todos ellos eran considerados funcionarios del Estado.

Aunque los informes de depuración podían abrirse de oficio, al convertirse la depuración positiva en un requisito imprescindible para trabajar en la Administración, lo habitual es que fuesen los propios interesados los que solicitaran la apertura del expediente. El número de solicitudes fue tan abundante que se produjo una gran demora en su tramitación. El Ministerio de la Gobernación dispuso que, con el fin de salvar tales dificultades, el Jefe Nacional de Sanidad pudiera delegar en los Inspectores Provinciales de Sanidad «la facultad de ordenar la práctica de nuevas diligencias y de proponer acuerdo en las informaciones de depuración de Médicos de Asistencia pública domiciliaria, Farmacéuticos, Practicantes y Matronas». En estos casos, la resolución de las informaciones quedaba delegada en los Gobernadores Civiles (BOE, 3 de agosto de 1939).

4. Rubio Cabeza, Manuel. Diccionario de la Guerra Civil española. Barcelona: Editorial Planeta; 1987, Vol. 1, p. 254. 
El personal sanitario, al igual que todo ciudadano, podía ser llevado ante los Tribunales de Responsabilidades Políticas (de ámbito nacional, regional, provincial), que constituyeron una compleja red de instancias represoras cuyo objetivo era que todas aquellas personas que hubieran formado parte del Frente Popular «reparasen los daños» derivados de la Guerra Civil. Con extraordinaria frecuencia, el encausado era sancionado económicamente o se procedía a la incautación y confiscación de sus bienes ${ }^{5}$. En el presente trabajo analizamos cómo el proceso de depuración política afectó al colectivo profesional de matronas que ejercían en Madrid, así como las diferencias que presenta con respecto a otros colectivos, como los médicos.

\section{La implicación de varios ministerios en la depuración de las matronas de Madrid}

Ya hemos comentado que el nuevo régimen convirtió en imprescindible la depuración positiva para regresar al puesto de trabajo o para acceder a la función pública. Esto explica que la persona objeto de la depuración se dirigiera a una instancia u otra para solicitarla, aunque, como ya hemos indicado, en algunos casos se abrían expedientes de oficio.

Así, la depuración de las matronas madrileñas que se habían incorporado en diferentes Departamentos de la Universidad Central a partir de los años veinte, dependería del juez instructor depurador del personal docente de la Universidad de Madrid y en último término del Ministerio de Educación Nacional; las que accedieron a las plazas de la «Sección Femenina Auxiliar del Cuerpo de Prisiones» durante la República, del juez depurador de las funcionarias de prisiones y, por tanto, del Ministerio de Justicia; y las que tuvieron dependencia directa de la Dirección General de Sanidad, del Ministerio de la Gobernación ${ }^{6}$. La depuración de las matronas que no tenían dependencia ministerial, entre ellas las que dependían de las corporaciones

5. Para conocer el funcionamiento de estos tribunales puede consultarse: Álvaro Dueñas, Manuel. Los militares en la represión política de la posguerra: la jurisdicción especial de responsabilidades políticas hasta la reforma de 1942. Revista de Estudios Políticos (Nueva Época). 1990; 69: 141-162.

6. Así, por ejemplo, la matrona de asistencia pública domiciliaria Elvira Prieto Ferrer fue separada definitivamente del servicio, por orden del Ministerio de la Gobernación de 27 de abril de 1943 (BOE, 13 de mayo de1943). 
locales, fue encomendada por la Dirección General de Sanidad, en octubre de 1940, a los Colegios de Médicos de las diferentes provincias ${ }^{7}$.

El Colegio de Médicos de Madrid, previamente, había iniciado la depuración de los médicos colegiados con anterioridad al 18 de julio de 1936, para lo que, cada uno de ellos, hubo de formular la correspondiente declaración jurada. El profesor Albarracín señaló como entre el 22 de abril de 1939 y el 9 de enero de 1940 el Colegio madrileño depuró favorablemente a 1.936 médicos $^{8}$. Lógicamente, como la mayoría de las matronas madrileñas no tenían dependencia ministerial, fue el Colegio de Médicos de Madrid el que llevó a cabo la depuración mayoritaria de las mismas; aunque lo que no estimamos tan lógico es que no fuera el propio Colegio de Matronas de Madrid el que depurara a sus asociadas. La explicación a esta circunstancia la podemos encontrar en la Real orden de 7 de mayo de 1930 (Gaceta de Madrid, 9 de mayo de 1930), en la que se concedía la colegiación obligatoria a las matronas y se aprobaban los estatutos para el régimen de los colegios. Dichos estatutos, suponían una legitimación por parte de los poderes públicos del sometimiento de las matronas al colectivo médico. Este sometimiento lo podemos considerar como el final del proceso de subordinación, lento pero inexorable, que habían emprendido siglos antes los médicos, como representantes de la autoridad patriarcal, contra el colectivo exclusivamente femenino formado por las matronas, a las que había que limitar sus competencias, enseñar y controlar. Los estatutos de los Colegios Oficiales de Matronas contemplaban que una de las funciones de estos era:

7. Sobre la depuración de las matronas en la provincia de Cádiz, se cuenta con el estudio de: Cabrera-Alfonso, Juan-Rafael; Herrera Rodríguez, Francisco. Expedientes de depuración de profesionales sanitarios a través de la documentación existente en el colegio oficial de médicos de Cádiz (1941-1946). In: Martínez-Pérez, José et al., eds. La medicina ante el nuevo milenio: una perspectiva histórica. Cuenca: Ediciones de la Universidad de Castilla La Mancha; 2004, p. 401-421. En el caso del Colegio Oficial de Médicos de Barcelona hay una referencia a la depuración de las matronas en: Cañellas, Cèlia. El Colllegi de Metges de Barcelona i la societat catalana del seu temps (1894-1994). Barcelona: Col.legi Oficial de Metges, 1994, p. 241. También hay referencias a la depuración de las matronas en la provincia de Almería en: Mirón González, Rubén. Depuración franquista del personal sanitario de la Beneficencia provincial de Almería [documento inédito]. XI Congreso Nacional y VI Internacional de Historia de Enfermería. Barcelona; 2010.

8. Albarracín Teulón, Agustín. Historia del Colegio de Médicos de Madrid. Madrid: Ilustre Colegio Oficial de Médicos de Madrid; 2000, p. 429-430. 
«Establecer y fomentar relaciones de concordia, siempre con la debida subordinación y disciplina, con los colegios médicos provinciales, para quienes estarán obligados a acatamiento y respeto».

Y no solo eso, las tarifas de honorarios que cada colegio de matronas debía establecer para los servicios más corrientes de la profesión, debían ser sometidas a examen y aprobación del colegio médico de la provincia correspondiente. No es de extrañar, pues, que fuesen los colegios médicos los encargados de depurar a las matronas.

Queremos resaltar que algunas de las matronas que ejercían la profesión al comienzo de la Guerra Civil no solicitaron la depuración después de la contienda. Probablemente algunas habrían fallecido, a otras no les interesaría continuar ejerciendo la profesión y cabe suponer, también, que aquellas que hubieran adquirido un mayor compromiso con el gobierno de la República, o que hubieran destacado por una mayor implicación sindical hasta la Guerra Civil, no solicitaran la depuración, bien por estar encarceladas, o por temer ser represaliadas, o bien por encontrarse fuera de Madrid. Incluso a algunas las hemos encontrado exiliadas fuera de España. Como de esto vamos a ocuparnos con detalle en un próximo trabajo, apuntaremos aquí, tan solo, los nombres de cinco matronas que no solicitaron la depuración porque estaban encarceladas: Soledad Ruiz Hernando, Margarita Pantigas Velázquez, Josefa Domenech Elizondo, Teresa Sancho Cervera y Ana Torres Gómez ${ }^{9}$. Una de las matronas más duramente perseguidas por la dictadura franquista fue Trinidad Gallego Prieto. No figura en la lista de colegiadas del Colegio Oficial de Matronas de Madrid de 1935 porque su título no fue expedido hasta el 22 de octubre de ese mismo año ${ }^{10}$. Tampoco solicitó la depuración después de la guerra, pues fue encarcelada tempranamente ${ }^{11}$. Con ocasión del 70 aniversario de la Guerra Civil, se publicaron en dife-

9. Todas ellas estaban en la lista del Colegio de Matronas de Madrid de 1935 con los números 343, 233, 431, 33 y 439 respectivamente. Lista de matronas, n. 3.

10. Expediente de Trinidad Gallego Prieto. Archivo General de la Facultad de Medicina de la Universidad Complutense de Madrid (en adelante AFMUCM), Secretaría de alumnos, Expedientes de matronas y practicantes, leg. 183, exp. 53.

11. Trinidad Gallego fue condenada a treinta años de prisión por sentencia de 27 de junio de 1939 acusada de un delito de «adhesión a la rebelión». Ingresó en la prisión de Amorebieta el 27 de agosto de 1939. Archivo General de la Administración (en adelante AGA), Justicia, Tratamiento Penitenciario 3,8_01005R.jpg. 
rentes medios de comunicación algunos testimonios sobre esta matrona, fallecida recientemente ${ }^{12}$.

\section{Depuración y sanción de las trabajadoras en la Universidad Central}

Hemos localizado seis mujeres que, teniendo la titulación de matronas, trabajaban en distintos departamentos de la Universidad Central en los años previos a la Guerra Civil y durante buen tiempo de ésta. Se trata de Asunción Muñoz Prieto, nombrada «masagista» (sic) del Hospital Clínico según R.O. de 3 de marzo de 1925룰 Marina Vera Aguilera, el 18 de julio de 1932, practicanta en el Departamento de Ginecología y Obstetricia de la Facultad de Medicina ${ }^{14}$; Josefina Cobos Martín, el 29 de agosto de 1932 auxiliar conservadora del Museo de Anatomía de la Facultad de Medicina, con un sueldo de 1.800 pesetas anuales ${ }^{15}$; María Teresa Arroyo Nieto, el 15 de febrero de 1933, ayudante sanitaria temporal encargada de la estadística del tracoma del Departamento de Oftalmología de la Facultad de Medicina ${ }^{16}$; Aurora Azpiri Lence y María Dolores Márquez Méndez, el 19 de diciembre de 1935, matronas del Departamento de Ginecología y Obstetricia de la Facultad de Medicina con un sueldo anual de 1.500 pesetas ${ }^{17}$. Las seis figuraban en la relación del Colegio de Matronas de Madrid de 1935, con los siguientes números de colegiadas: 271, 88, 1, 318, 435 y 309. Como ya hemos señalado, la doble titulación podía considerarse una ventaja a la hora de acceder a un puesto de trabajo. Es el caso de Marina Vera Aguilera, quien, aunque estaba contratada como practicanta, en los años de la posguerra atendió multitud de partos en el Hospital Clínico ${ }^{18}$.

12. Trinidad Gallego. Disponible en: http://www.elmundo.es/especiales/2006/07/espana/guerracivil/hist_gallego.html. Un reportaje histórico, en el que Trinidad Gallego Prieto, de 95 años, cuenta cómo vivió la cárcel durante la Guerra Civil. Disponible en: http://elviajero. elpais.com/audios/espana/Memorias/matrona/Guerra/Civil/elpaudnac/20090401csrcsrnac_8/ Aes/ . Trinidad Gallego prieto. Disponible en: http://enmemoria.lavanguardia.com/obituarios/ trinidad-gallego-prieto.html.

13. AGA, Educación 21/29391.

14. AGA, Educación 21/20394.

15. AGA, Educación 21/20387.

16. AGUCM, P-0441-75.

17. AGUCM, P-0442-28 y P-0585-8.

18. AGUCM, P-0735-33. 
$\mathrm{Al}$ acabar la Guerra Civil, siendo rector de la Universidad de Madrid el catedrático de Historia Moderna y Contemporánea Pío Zabala y Lera, es nombrado decano de la Facultad de Medicina Fernando Enríquez de Salamanca y Danvila, catedrático de Patología y Clínica Médica. Poco después sería nombrado juez instructor del Tribunal de Depuraciones Políticas del profesorado de la Universidad de Madrid. Su elección no fue aleatoria, puesto que tanto su conocimiento interno de la Universidad y de su personal, como el haber sido él mismo depurado por la República al inicio de la guerra, lo convertían en la persona idónea para realizar esta función. Enríquez de Salamanca, llamado por muchos «el Inquisidor», procedió a formar expedientes de depuración al profesorado y demás personal dependiente de la Universidad, mediante la aplicación de la Ley de 10 de febrero de 1939. Entre los documentos que comprendía un expediente se encontraba la declaración jurada del interesado y la solicitud de informes socio-políticos a curas párrocos, a la Guardia Civil y a las Jefaturas locales de Falange. Además, cualquier ciudadano (vecino, compañero de trabajo, etc.) podía aportar su juicio, positivo o negativo, sobre la persona que se tratara ${ }^{19}$.

Presentaremos como ejemplo-tipo, el expediente que se formó a Josefina Cobos Martín y que se conserva en el Archivo General de la Administración ${ }^{20}$. El mismo consta de una declaración jurada de la interesada (3 de abril de 1939), la solicitud mecanografiada de readmisión en el cargo (13 de abril), la declaración jurada, también mecanografiada, a la que aludía el Decreto de 21 de enero de 1939 (13 de abril), un impreso del Juzgado Militar de Funcionarios por el que se reclaman informes sobre Josefina Cobos al Alcalde de Madrid (26 de mayo, cumplimentado con las respuestas el 19 de octubre), otra declaración de la interesada donde se ratifica de lo dicho por medio de su declaración jurada (24 de julio), sendos certificados de Edelmiro Sardinero Rey, de la 2. ${ }^{a}$ Centuria de la Agrupación de Centurias

19. Pérez Peña, Fernando. Exilio y depuración política en la Facultad de Medicina de San Carlos (sus profesores y la Guerra Civil). Madrid: Editorial Vision Net; 2005, p. 61-66. Pueden consultarse los siguientes trabajos sobre la influencia de la represión franquista en la Universidad: Otero Carvajal, Luis Enrique et al. La destrucción de la ciencia en España. Madrid: Editorial Complutense; 2010; Claret Miranda, Jaume. El atroz desmoche. La destrucción de la universidad española por el franquismo, 1936-1945. Barcelona: Crítica; 2006, Rodríguez López, Carolina. La Universidad de Madrid en el primer franquismo: ruptura y continuidad (1939-1951). Madrid: Dykinson; 2002.

20. AGA, Educación 21/20387. 
de Madrid (26 de julio) y de José María Palacios Porta, excombatiente en el Ejercito Nacional como voluntario (26 julio) y de la propuesta de readmisión del juez instructor (1 de agosto).

La declaración jurada se formulaba sobre un impreso en el que, después de completar los datos personales (en su caso nos informa que contaba 46 años, era viuda y natural de Zaragoza), se respondía a trece preguntas. Entre ellas figuraban la fecha de ingreso en la administración (septiembre de 1928), el sueldo (1.800 pesetas), si había ascendido con posterioridad al 18 de julio (no), si había estado afiliada a alguna organización sindical (UGT desde enero de 1937), si había formado parte de algún batallón de milicianos (no) y si había formado parte del algún comité de salud pública o junta depuradora de funcionarios (no). A la pregunta más comprometedora, la octava, que llevaba por enunciado «Diga quiénes eran los más destacados izquierdistas de su departamento y cuanto sepa de la actuación de los mismos», responde: «D. Manuel Herrero, afiliado a Izquierda Republicana. Ignoro su actuación» ${ }^{21}$. Con respecto a la delación, hay que decir que existían diversas estrategias para contestar a la pregunta sin que se tratase de una denuncia formal. Una, consistía en nombrar a una persona que se sabía en el exilio. Otra, la que usó Josefina Cobos, era nombrar a alguien de quien era ya conocida su afiliación política.

Por su parte, en la declaración jurada a la que aludía el Decreto de 21 de enero de 1939, debía de darse respuesta a veinte preguntas muy similares a las ya apuntadas en el impreso anterior ${ }^{22}$, si bien se preguntaba expresamente por la pertenencia a partidos políticos, a la masonería, y a las milicias del Frente Popular, por la residencia en el extranjero o «en población dominada por el enemigo» así como por el nombre de las personas que podían confirmar las manifestaciones del declarante (por lo menos dos) y por las

21. Manuel Herrero Benítez (1900-1979), Ayudante de anatomía en la Facultad de Medicina, estuvo afiliado a Izquierda Republicana y a la Agrupación de Médicos Liberales, siendo capitán médico durante la guerra. El profesor Francisco Guerra señala que fue sancionado por el Colegio de Médicos de Madrid, el 22 de junio de 1942, a la inhabilitación perpetua para desempeñar cargos directivos de confianza y cesado como docente por el Tribunal Universitario de Responsabilidades Políticas. Guerra, Francisco. La medicina en el exilio republicano. Madrid: Universidad de Alcalá; 2003, p. 155.

22. Comenzaba diciendo: «El que suscribe jura por Dios y por su honor decir la verdad a las preguntas que comprende el siguiente interrogatorio y quedando apercibido de las responsabilidades tanto de orden administrativo como criminal en que quedaría incurso si por mala fe o cualquiera otra circunstancia omitiera o falseara algunos hechos, que serían juzgados por el fuero de guerra». 
pruebas documentales que podía aportar. Señalar, tan solo, que Josefina Cobos apuntaba como personas que podían confirmar sus manifestaciones a Julián de la Villa Sanz, domiciliado en Marqués del Duero 4, y Rodrigo Díaz Guerra, del que apunta que es «agente del S.I.P.M.» ${ }^{23}$.

La instrucción completa del expediente de depuración se prolongaba durante tres o cuatro meses, si este se desarrollaba con normalidad. Por la misma orden, de 25 de octubre de 1939 (BOE, 08/11/1939), por la que se rehabilitaba sin imposición de sanción a Josefina Cobos, se hacía lo propio con Aurora Azpiri Lence y M. ${ }^{a}$ de la Asunción Muñoz Prieto. La orden iba firmada por José Ibáñez Martín, ministro de Educación Nacional. Aurora Azpiri había nacido en Bilbao, el 3 de julio de 1882 y había seguido los estudios de matrona en Madrid en los cursos 1920-21 y 1921-22 ${ }^{24}$. Solicitó la depuración el 5 de mayo de 1939. Asunción Muñoz Prieto era de Madrid, donde nació el 15 de agosto de 1892. Había obtenido la calificación de sobresaliente en ambas reválidas de matrona y practicanta, y sobresaliente con Matrícula de Honor en los dos cursos de matrona, en la Universidad Central $^{25}$.

Marina Vera Aguilera, de la que ya hemos señalado que era practicanta en el Departamento de Ginecología y Obstetricia, había sido rehabilitada con anterioridad, concretamente por orden del 4 de septiembre (BOE, 18/09/1939). A su favor, sin duda, la certificación de Caya Eugenia Lasterra Vidaurre, organizadora de «Auxilio Social» de Madrid de:

"Que Marina Vera Aguilera la pertenecido a la Organización clandestina AUXILIO SOCIAL desde el primer momento trabajando en ella con verdadero entusiasmo en cuantas misiones le encomendaron sus Jefes por difíciles y expuestas que estas fueran ${ }^{26}$.

23. El Servicio de Información y Policía Militar (SIPM) fue un organismo creado en zona de dominación franquista, a finales de 1937, con objeto de coordinar todas las actividades encaminadas a la información, el espionaje y el contraespionaje en la zona republicana.

24. AGA, Educación 32/15098.

25. AGA, Educación 32/14385.

26. AGA, Educación 21/20394. 


\subsection{M. ${ }^{a}$ de los Dolores Márquez Méndez}

Diferente fue lo sucedido con M. ${ }^{a}$ de los Dolores Márquez Méndez. Aunque solicitó su depuración el día 25 de abril de $1939^{27}$, no se presentó ante el juez instructor. Nacida en Madrid el 9 de abril de $1902^{28}$, había cursado sus estudios en la Universidad Central durante los cursos académicos 1927-28 y 1928-29, realizando las prácticas en el Hospital Provincial y en el Hospital Clínico respectivamente. Su título fue expedido con fecha 11 de enero de 1930. Durante la Guerra Civil fue vicepresidenta del Colegio Oficial de Matronas de Madrid, del que se había incautado la Agrupación de Matronas de Madrid y Limítrofes, organización afecta a la UGT a la que pertenecía ${ }^{29}$. Enríquez de Salamanca, todavía como decano de la Facultad de Medicina, en oficio de 22 de enero de 1940 comunicaba al Rector que la matrona "ha cesado en el desempeño de dicho cargo con fecha 31 de Diciembre próximo pasado por haber abandonado el servicio sin haber sido posible precisar su paradero». Esto motivó que, por orden de 4 de marzo del Ministerio de Educación Nacional (BOE, 18/03/1940), se la separase del servicio. A pesar de ello, a primeros de abril, el juez instructor la citó para que, en el plazo de diez días, compareciera en su Juzgado, sito en el Decanato de la Facultad de Medicina, a fin de deponer en el expediente formal que se instruía contra ella ${ }^{30}$. Al no presentarse, Enríquez de Salamanca comunicó al Rector, el 28 de abril de 1940, que:

«con esta fecha y después de instruido el expediente formal a la Matrona de la Facultad de Medicina de esta Universidad D. a María Dolores Márquez Méndez, propongo al Excmo. Sr. Ministro de Educación Nacional, la separación definitiva del servicio».

Finalmente, por orden de 21 de mayo de 1940 (BOE, 26/06/1940), el Ministerio resolvió su separación definitiva del servicio, de conformidad con la propuesta formulada por el juez instructor y a tenor de ordenado

27. Archivo General de la Universidad Complutense de Madrid (en adelante AGUCM), SG-1300.

28. Su padre, Sebastián Márquez Pérez, de estado soltero, era jornalero. Su madre Dolores Méndez Hernández era viuda. AGA, Educación 32/14568.

29. Incautación del Colegio Oficial de Matronas de Madrid. ABC. 31 Jul 1936: 30.

30. Edicto. ABC. 7 Abr 1940: 20. 
por la «Ley de 10 de febrero de 1939 y lo dispuesto en la Orden de 12 de marzo del mismo año» ${ }^{31}$.

\subsection{María Teresa Arroyo Nieto}

El caso de María Teresa Arroyo Nieto fue distinto también. Era natural de Madrid, donde nació el 29 de marzo de 1902 y donde siguió los estudios de matrona en la Universidad Central. El primer curso, en 1927-28, hizo las prácticas en el Hospital Provincial, y el segundo, en 1928-29, las hizo en el Hospital Clínico ${ }^{32}$. En 1933 fue nombrada ayudante sanitaria temporal, encargada de la estadística del tracoma del Departamento de Oftalmología, empleo por el que cobraba 2.000 pesetas anuales. Dicho departamento lo dirigía el doctor Manuel Márquez, que ese mismo año organizaba en Madrid el XIV Congreso Internacional de Oftalmología.

El levantamiento militar la sorprendió estando en El Espinar ${ }^{33}$, desde donde el Socorro Rojo la llevó a Francia, a casa de unos familiares, y posteriormente a Barcelona, donde se había trasladado la mayor parte del Departamento de Oftalmología de la Universidad Central y donde presentó su adhesión al gobierno de la República. Lógicamente, no solicitó su depuración. Posteriormente emigró a México, ingresando por Nuevo Laredo el 19 de noviembre de 1939 como asilada política ${ }^{34}$.

En México siguió colaborando con el Dr. Manuel Márquez y con su esposa, la Dra. Trinidad Arroyo, una de las primeras oftalmólogas españolas ${ }^{35}$. Tras la muerte de la Dra. Arroyo, en otoño de 1959, prosiguió la estrecha colaboración de Teresa Arroyo con su mentor ${ }^{36}$.

31. Orden de 21 de mayo de 1940 por la que se separa definitivamente del servicio a la Matrona de la Facultad de Medicina de Madrid doña María de los Dolores Márquez Méndez. BOE 26 de junio de 1940.

32. AGA, Educación 32/14564.

33. AGA, Educación 31/1047.

34. María Teresa Arroyo. Registro Nacional de Extranjeros en México. Copia digital en: Ministerio de Cultura. Movimientos migratorios iberoamericanos. http://pares.mcu.es/MovimientosMigratorios/

35. Márquez Arroyo, Cristina. Trinidad Arroyo de Márquez (1872-1959): primera oftalmóloga española, políglota, redactora médica y una mujer de armas tomar. Panace@ [artículo en internet].2010 [citado 1 Jun 2011]; XI: 31. Disponible en: http://www.tremedica.org/panacea.html

36. Rivas Cherif, Manuel de. El Dr. Manuel Márquez. Su vida y su personalidad científica. Ciencia. 1962; 22 (1-2): 1-16 (16). 


\section{Depuración y sanción de las matronas que actuaron como funcio- narias de prisiones}

Victoria Kent fue la Directora general de Prisiones desde el 18 de abril de 1931 (Gaceta de Madrid, 19 de abril de 1931) hasta el 4 de junio de 1932 (Gaceta de Madrid, 8 de junio de 1932), fecha en que fue admitida su dimisión ${ }^{37}$. Durante el escaso periodo de tiempo que estuvo a su cargo el sistema penitenciario, emprendió una ambiciosa tarea de reforma de éste, con la finalidad de humanizarlo y modernizarlo. Entre otras medidas, como el aumento en el presupuesto para la ración alimenticia de los reclusos (Gaceta de Madrid, 13 de mayo de 1931) y la eliminación del uso de cadenas y grilletes en las prisiones (Gaceta de Madrid, 14 de mayo de 1931), impulsó la creación de la «Sección Femenina Auxiliar del Cuerpo de Prisiones», que se materializó en el decreto de 23 de octubre de 1931 (Gaceta de Madrid, 24 de octubre de 1931). Entre las funciones de la nueva sección se encontraban la vigilancia y custodia de las reclusas en la Prisión Central de Mujeres de Alcalá de Henares, el Reformatorio de Mujeres de Segovia y las Prisiones Provinciales de Mujeres de Madrid, Barcelona y Valencia. Además, con la supervisión del responsable de la dirección y administración de cada establecimiento, debían encargarse de la instrucción y educación de las presas, sustituyendo así a las Hijas de la Caridad, que hasta entonces habían desarrollado las labores de vigilancia de las reclusas.

Los requisitos que se solicitaban para acceder a dichas plazas consistían en contar con una edad comprendida entre los veintisiete y los cuarenta y cinco años, y tener conocimientos de cultura general, nociones de gramática, geografía, historia y aritmética, y se especificaba que tendrían preferencia aquellas que presentasen algún título facultativo o acreditasen el conocimiento de algún oficio de especial aplicación a las actividades de la mujer. Como podemos observar, a pesar de que la época republicana supuso un

37. El cargo de Director General de Prisiones no debía ser sencillo de desarrollar: desde la dimisión de Victoria Kent, en junio de 1932, hasta diciembre de 1933 cuatro personas más pasaron por el mismo: Vicente Sol Sánchez (nombramiento y dimisión respectivamente en Gaceta de Madrid, 8 de junio de 1932 y 29 de abril de 1933), Manuel Ruiz Maya (nombramiento y dimisión respectivamente en Gaceta de Madrid 7 de mayo de 1933 y 14 de septiembre de 1933), José Estellés Salarich (nombramiento y dimisión respectivamente en Gaceta de Madrid 14 de septiembre de 1933 y 3 de diciembre de 1933) e Hipólito Jiménez y Jiménez Colorado (nombramiento en Gaceta de Madrid 24 de diciembre de 1933). 
avance en la participación femenina en distintas esferas, todavía existía una amplia dicotomía entre los roles masculino y femenino.

Para ser admitidas a dicho concurso de provisión, convocado por primera vez con fecha 26 de octubre de 1931 (Gaceta de Madrid, 27 de octubre de 1931), y en el que se ofertaban 34 plazas, las aspirantes debían presentar su instancia firmada y dirigida a la Directora General de Prisiones acompañada de los documentos siguientes: una certificación de nacimiento, una certificación negativa del Registro de Penados y Rebeldes, un certificado de buena conducta y cualquier otro acreditativo de los méritos de aplicación al carácter y misión de este cargo.

Antes de cumplirse el plazo de presentación de instancias, se publicaron las normas para el desarrollo de las oposiciones (Gaceta de Madrid, 13 de noviembre de 1931), entre las que se especificaban las pruebas a las que serían sometidas las concursantes, si bien aquellas que acreditasen poseer un título de Facultad universitaria, como era el caso de las matronas, de Escuela Normal, de Instituto de Segunda Enseñanza o de Escuela de Comercio, no debían someterse a la prueba inicial de conocimientos. El segundo ejercicio, al que concurrirían tanto aquellas aptas en el primer ejercicio como las tituladas, consistía en el desarrollo por escrito de cuestiones de índole penal y pedagógica relacionadas con el régimen penitenciario. Tras este segundo ejercicio, el Tribunal designado elevaría la propuesta de las declaradas aptas para continuar en el proceso. Éstas, tenían la obligación de asistir a un «cursillo especial de conocimientos penitenciarios» de dos meses de duración.

A la finalización de dicho cursillo, que tendría lugar en los locales de la Escuela de Criminología anexa a la Prisión Celular de Madrid, los cuatro profesores encargados de impartirlo propondrían la lista de opositoras que deberían pasar a los ejercicios prácticos. Tras la realización de dichas prácticas, se formaría una lista definitiva de aprobadas por riguroso orden de méritos. Estamos de acuerdo con Gloria Ángeles Franco Rubio, cuando afirma que:

«esta sección era un cuerpo muy especializado, con unas pruebas de aptitud severas y una preparación superior a lo normal, para las que la mayoría de las mujeres españolas no estaba preparada ni con posibilidades de estarlo al menos de momento» ${ }^{38}$.

38. Franco Rubio, Gloria Ángeles. La incorporación de la mujer a la administración del Estado, municipios y diputaciones. Madrid: Subdirección General de Estudios e Investigaciones, Dirección General de la Juventud y Promoción Sociocultural; 1981, p. 54. 
Hemos identificado un buen número de matronas madrileñas en el listado de las 529 aspirantes que se publicó en la Gaceta del día 16 de noviembre de 1931. De ellas, nombraremos solo a unas pocas, en concreto a Emilia Adrián Romero, Ana Domínguez Villalba, Encarnación Melgares Mateos, Dolores Márquez Méndez y Felicidad Sáez Hernández, que serían sancionadas posteriormente por el Colegio de Médicos de Madrid y otras instancias. El número de candidatas se redujo sustancialmente después de la primera criba que supuso los exámenes previos, de tal forma que finalmente fueron 101 las opositoras seleccionadas para realizar el cursillo antes mencionado $^{39}$. Finalizado éste, solo quedaban 56 aspirantes para la realización de las pruebas prácticas que tendrían lugar en la Prisión de Mujeres de Madrid y en la Central de Alcalá de Henares, y que se iniciaron el 11 de abril de 1932 (Gaceta de Madrid, 6 de abril de 1932).

Curiosamente, no aparece en la Gaceta el nombramiento oficial de las nuevas funcionarias, y hay que recurrir al escalafón de $1935^{40}$ para comprobar cuáles de ellas accedieron finalmente a los puestos de trabajo. En dicho escalafón, figuraban: en el número cinco Purificación de la Aldea y Ruiz de Castañeda, en el número seis Áurea Rubio Villanueva, ambas dentro de la categoría de oficiales con tres mil quinientas pesetas, y como aspirante a oficial, Catalina Mayoral Arroyo. Como veremos más adelante, tanto Purificación de la Aldea como Catalina Mayoral aparecen en la lista del Colegio Oficial de Matronas de Madrid de 1935, sin embargo no aparece en dicha relación Áurea Rubio Villanueva, debido a que había realizado una permuta de su plaza original en Madrid por otra en Valencia con Dolores Freixa Batlle ${ }^{41}$.

Las ya nombradas funcionarias, presentaron una instancia solicitando que se sustituyese la denominación de «Auxiliares» por la de «Oficiales» (Gaceta de Madrid, 17 de septiembre de 1932), adecuándose a su equivalente masculino de los Establecimientos de reclusión de hombres. Dicha solicitud, que fue aceptada por el entonces ministro de Justicia Álvaro de Albornoz, estaba suscrita en primer lugar por Áurea Rubio Villanueva, una de las matronas que consiguieron plaza en la oposición. Esto muestra su

39. La relación de admitidas al cursillo aparece en la Gaceta de Madrid, 23 de diciembre de 1931.

40. Los datos del mencionado escalafón se han obtenido de la obra de Franco Rubio, n. 38, p. 179-181.

41. Permuta. Vida Penitenciaria. 20 Oct 1935, p. 14. 
carácter inconformista, contribuyendo, dentro de sus posibilidades, a la lucha por la igualdad de derechos de las mujeres.

\subsection{Purificación de la Aldea y Ruiz de Castañeda}

Pura de la Aldea, como era conocida familiarmente, había nacido en Madrid el 10 de agosto de 1889. Estudió ambos cursos de la carrera de matrona en la Universidad Central, en el año académico 1921-22, y su título se expidió con fecha 4 de junio de 1924. También estudió la carrera de practicanta cuyo título había sido expedido el 14 de julio de $1923^{42}$. En 1935 figuraba con el número 20 de colegiada en el Colegio de Matronas de Madrid. Desarrolló su labor de funcionaria en la Prisión de Ventas, desde la inauguración de ésta hasta su cese, pasando a ser, como comenta Fernando Hernández Holgado, una "carcelera encarcelada» ${ }^{43}$. Este mismo autor destaca el gran recuerdo que dejó en las mujeres que la conocieron ${ }^{44}$.

Al margen de la depuración como funcionaria de prisiones, el Consejo de Guerra Permanente número tres, constituido en Madrid y por el procedimiento sumarísimo de urgencia, con fecha 1 de mayo de 1939 la condenó «como autora de un delito de adhesión a la rebelión sin circunstancias modificativas de la responsabilidad criminal a la pena de reclusión perpetua hoy TREINTA AÑOS de reclusión mayor y accesorias de inhabilitación absoluta». Entre los cargos que se consideraban probados figuraba su significación izquierdista y el hecho de que, como funcionaria de prisiones, había sido designada «durante la dominación roja» jefe de servicios de la prisión de Ventas. También se incluían como agravantes su dureza en el trato a las reclusas de ideas derechistas, su afiliación al PCE en 1936, su pertenencia a los Amigos de la Unión Soviética y el uso de una pistola durante el servicio ${ }^{45}$.

\footnotetext{
42. AGA, Educación 32/14379.

43. Hernández Holgado, Fernando. Carceleras encarceladas. La depuración franquista de las funcionarias de Prisiones de la Segunda República. Cuadernos de Historia Contemporánea [artículo en Internet]. 2005; 27: 271-290. Disponible en: http://revistas.ucm.es/ghi/0214400x/articulos/ CHCO0505110271A.PDF [citada 14 Jun 2011].

44. Hernández Holgado, Fernando. Mujeres encarceladas. La prisión de Ventas: de la República al franquismo, 1931-1941. Madrid: Marcial Pons Historia; 2003, p. 193-194.

45. Centro Documental de la Memoria Histórica (en adelante $(D M H)$, Tribunal de Orden Público 42/02663.
} 
Vista la sentencia condenatoria firme, y según se recogía en el artículo noveno de la ley 10 de febrero de 1939 (BOE 14 de febrero de 1939), fue separada definitivamente del servicio por orden 3 de julio de 1939 (BOE 7 de agosto de 1939). Cumplió parte de su condena en la prisión de mujeres de Barcelona y fue puesta en libertad condicional el 11 de julio de 1944, según consta en su ficha penitenciaria ${ }^{46}$. Ese mismo año, en la Audiencia Provincial de Madrid, se siguió expediente contra ella por responsabilidades políticas, si bien al considerarse que su patrimonio no rebasaba la cantidad de veinticinco mil pesetas ${ }^{47}$, que era la que el artículo 8 de la Ley de 19 de febrero de 1942 eximía de sanción, se decretó el sobreseimiento del expediente y archivo sin declaración de responsabilidad política ${ }^{48}$.

\section{2. Áurea Rubio Villanueva}

Había nacido en 1895 en la localidad de Herrera de Pisuerga (Palencia), hija de Jesús Rubio Coloma, abogado, y de Áurea Villanueva González. Era una mujer con una amplia formación, ya que además de haber realizado los estudios de matrona y practicanta, tenía el título de maestra de primera enseñanza del que se había revalidado el 27 de junio de $1913^{49}$. Realizó los dos cursos de la carrera de matrona durante el año académico 1924-25 para los que había sido necesaria la autorización de su marido Manuel de Lara Gil.

Fue la primera presidenta de la Agrupación de Matronas de Madrid y Limítrofes ${ }^{50}$, organización afecta a la UGT y que tenía su sede en la Casa del Pueblo. Muy probablemente estos antecedentes socialistas pesaron negativamente durante el curso de su depuración.

Como ya hemos mencionado, formó parte de la primera promoción de funcionarias de prisiones y tenía el número 6 del escalafón en 1935, año en

46. AGA, Justicia, Tratamiento Penitenciario 1,3_01288R.jpg. En su ficha penitenciaria aparece como divorciada, y sin domicilio conocido. Su ex marido se llamaba Luis March y Canals, según consta.

47. CDMH, Responsabilidades Políticas 75/00667.

48. $\mathrm{CDMH}$, Responsabilidades Políticas 75/00667.

49. AGA, Educación 32/14393.

50. Constitución de la Agrupación de Matronas de Madrid y Limítrofes. El Heraldo de Madrid. 2 Jul 1931; 14.170: 6 . 
el que realizó la permuta a Valencia ${ }^{51}$. En mayo de 1936 realizó la petición de ser nombrada comadrona en la prisión de mujeres de Valencia ${ }^{52}$, a lo que el Director General de Prisiones de Valencia accedió, matizando que no podría asignársele remuneración hasta que la consignación presupuestaria lo permitiese.

Fue separada definitivamente del servicio, a propuesta del instructor, con fecha 27 de julio de 1939 (BOE 2 de agosto de 1939). Según afirma Hernández Holgado, emigró a México donde falleció en 197953 .

\section{3. Catalina Mayoral Arroyo}

Nacida en Madrid el 9 de noviembre de 1901, estudió los dos años de la carrera de matrona durante los cursos académicos 1923-24 y 1924-25. En este último año inició sus estudios de practicanta, finalizándolos el curso siguiente con unas excelentes calificaciones en ambos $\operatorname{casos}^{54}$. Figuraba la número 131 en la lista del Colegio Oficial de Matronas de Madrid.

Si bien aparecía como aspirante a oficial del cuerpo femenino de prisiones en el escalafón de 1935, con fecha 8 de julio fue nombrada auxiliar del cuerpo de prisiones de la ciudad de Valencia. Fue la matrona que atendió el primer parto de la nueva prisión de mujeres de Madrid ${ }^{55}$. En 1935, aparece también como perteneciente al escalafón del Cuerpo de Instructoras de Sanidad con el número $28^{56}$.

En octubre de 1937, el director de la prisión de mujeres de Madrid le comunicó que su petición de aumento de remuneración a ciento cincuenta pesetas mensuales, en atención al excesivo trabajo realizado en la prisión

51. Este traslado posiblemente sea la causa de que no figure en la lista del Colegio Oficial de Matronas de 1935. Lista de matronas, n. 3.

52. CDMH, PS-MADRID 673,12.

53. Hernández Holgado, n. 43, p. 285.

54. AGA, Educación 32/14393.

55. La nueva prisión había sustituido al antiguo edificio de la calle Quiñones. El parto se produjo a la media hora de inaugurado el centro, por lo que el famoso fotógrafo Alfonso inmortalizó a Catalina Mayoral sosteniendo al recién nacido: Mundo Gráfico. 11 Oct 1933: 20. Fotos del mismo acontecimiento pueden consultarse en AGA (02) 035128, (02) 035129, (02) 035130 y (02) 035131.

56. La Visitadora Sanitaria, Madrid. 1935; 2 (5): 13. 
como "profesora en partos», había sido aceptada y comenzaría a consignarse desde ese mismo mes ${ }^{57}$.

Según el certificado firmado el 28 de noviembre de 1941 por Antonio Carrasco Cobo, secretario del Tribunal Regional de Responsabilidades Políticas de Madrid, fue dada de baja en el escalafón del cuerpo de prisiones por encontrarse en paradero ignorado o en el extranjero ${ }^{58}$. Un documento posterior, fechado concretamente el 2 de junio de 1945, decreta el sobreseimiento provisional del expediente, dado que la única acusación contra ella era que no había comparecido a la depuración. No deja de sorprendernos que la no comparecencia fuese la única acusación contra ella, teniendo en cuenta que esta estaba motivada por su encarcelamiento en la prisión de Ventas, la misma cárcel en la que había trabajado como funcionaria. Esto pone en evidencia la falta de coordinación existente en esos momentos entre los diferentes órganos represores. Fue condenada a doce años y un día por un delito de auxilio a la rebelión, debido a su doble afiliación a la UGT y al partido comunista durante el periodo republicano y, según señala la sentencia, por haber puesto espías para perseguir a médicos y enfermeras de derechas, lo cual pudo realizar desde su cargo de delegada de asistencia social e inspectora de higiene infantil. Como circunstancia atenuante se anotaba que no se portó mal con las detenidas ${ }^{59}$. Durante su época de reclusión en el presidio en Ventas se encargó de la enfermería de partos $^{60}$. En los últimos años vivió en Barcelona ${ }^{61}$. Sus restos descansan en el Cementerio de Montjuic ${ }^{62}$.

\section{La depuración de las matronas por el Colegio de Médicos de Madrid}

Ya hemos adelantado como, en el mes de octubre de 1940, se encomendó al Colegio de Médicos de Madrid la depuración de las matronas madrileñas

\footnotetext{
57. CDMH, PS-MADRID 673,12.

58. CDMH, Tribunal Orden Público 42/2813.

59. CDMH, Responsabilidades Políticas 75/00242.

60. Domingo, Carmen. Coser y cantar. Las mujeres bajo la dictadura franquista. Barcelona: Lumen; 2007, p. 190.

61. En 1962 solicitó al decano de la facultad de Medicina de Madrid el envío de su título de practicanta, que debía ser remitido a su domicilio en la calle Travesía de Gracia n. 367, 4.0-1. de Barcelona. AFMUCM, Secretaría de alumnos, Expedientes de matronas y practicantes, leg. 344, exp. 65.

62. Institut Municipal dels Serveis Funeraris de Barcelona. La Vanguardia. 30 Ene 1993: 53.
} 
y como fue éste quien más expedientes resolvió. Fue en la sesión celebrada por el Consejo Directivo del Colegio el día 17 de ese mes, cuando quedó formalmente enterado de la obligación que le incumbía de efectuar la depuración de las matronas de Madrid. En dicha sesión, presidida por Guillermo Núñez y con la asistencia de Calixto González de Quevedo, Jesús del Pino, Manuel Alonso Canalejas y Antonio Utrilla, éste último actuando como secretario $^{63}$, se tomó el acuerdo de citar a la presidenta del Colegio de Matronas para disponer lo necesario al efecto ${ }^{64}$.

Casi cinco meses después, el día 12 de marzo de 1941, el Pleno del Consejo Directivo Provincial del Colegio, en sesión presidida por José Fernández de la Portilla, que era a su vez el presidente del Colegio de Médicos, aprobó la depuración sin sanción alguna, con arreglo a los antecedentes recibidos, de las primeras 185 matronas ${ }^{65}$. De ellas, algo más de las dos terceras partes, en concreto 126, figuraban en la lista de colegiadas elaborada por el Colegio Oficial de Matronas de Madrid en 1935.

En las actas de las sesiones celebradas en los meses siguientes, tanto por el Consejo Directivo, como por el Pleno del Consejo Directivo del Colegio, queda constancia de las matronas que fueron quedando depuradas sin sanción, en cada una de ellas: 57 en la sesión de la Comisión del 14 de abril; 29 en la del día 19 de septiembre, si bien una de ellas, Elisa Dorado Llanera, sería sancionada posteriormente por el propio Colegio; una por el Pleno del 18 de octubre; dos por el del 18 de noviembre; cinco en el de 29 de noviembre y, así, en sesiones sucesivas, hasta la Comisión Permanente celebrada el día 19 de junio de 1942 en la que, junto a la depuración sin sanción de cuatro matronas, se acordó la devolución de los expedientes de otras seis (Consuelo Garrido Martínez, Paula Encinas Polanco, Encarnación García Fernández, Antonia Mora Serrano, Felicidad Sáez Hernández y Elvira Iglesias Pozo), por ignorarse su paradero y hasta tanto que, en forma reglamentaria, solicitaran su nueva depuración. Sabemos que Paula Encinas Polanco, había sido sentenciada a la pena de seis años y un día de reclusión mayor por el Consejo de Guerra permanente n. 8 reunido en la

63. Estos cinco médicos, junto a José Fernández de la Portilla (presidente) y Eduardo Isla Carande, integraban la Junta Directiva del Colegio de Médicos de Madrid que, bajo la presidencia del gobernador civil (José Finat y Escrivá de Romaní, conde de Mayalde) y ante el director general de Sanidad, habían tomado posesión el 12 de enero de 1940. Toma de posesión de la Junta del Colegio de Madrid. ABC. 13 Ene 1940: 11.

64. Libro de Actas del Colegio de Médicos de Madrid, f. 241 [17 Oct 1940].

65. Libro de Actas del Colegio de Médicos de Madrid, f. 18v.-20v. [12 Mar 1941]. 
Plaza de El Escorial, el 26 de agosto de 1939, por un delito de excitación a la rebelión ${ }^{66}$. Seis años después, dos de ellas, Consuelo Garrido y Felicidad Sáez, serían inhabilitadas por el Colegio de Médicos ${ }^{67}$. Solo hubo un caso de expediente abierto de oficio por el juez instructor: fue el de Antonia Álvarez Martínez, que fue sancionada con inhabilitación para ocupar cargos de confianza y directivos.

Teniendo en cuenta que en el artículo cuarto de la ley 10 de febrero de 1939, se recogía que los instructores comenzarían su labor por los casos en que fuese más patente la adhesión al «Movimiento Nacional», resulta comprensible que fueran retrasando la resolución de aquellos expedientes susceptibles de sanción. Las primeras sanciones se produjeron en la sesión plenaria de la Junta de Gobierno celebrada el día 22 de junio de 1942. En ella, la depuración de cuarenta y dos matronas se tradujo en sanciones diversas: inhabilitación para ocupar cargos de confianza y directivos y periodos de privación del ejercicio profesional, en la jurisdicción del Colegio Oficial de Matronas de la provincia de Madrid, que oscilaban entre seis meses y tres años. Sin embargo no se produjeron sanciones de traslado forzoso ni de separación definitiva del servicio. Estas cuarenta y dos matronas sancionadas el 22 de junio de 1942 se han recogido en la TABLA 1, junto con las que serían sancionadas en años posteriores.

El Colegio de Médicos de Madrid, hasta el día 22 de junio de 1942, había resuelto 367 expedientes de depuración con el siguiente resultado: 319 expedientes favorables (de estos, 187 correspondían a matronas que figuraban en la lista de colegiadas de 1935); 42 expedientes finalizaron con sanción (34 de ellas estaban colegiadas en Madrid en 1935); y 6 expedientes fueron devueltos (3 de ellos de las matronas de la lista de 1935). Si lo analizamos globalmente, el 61\% de los expedientes instruidos por el Colegio de Médicos, hasta esa fecha, correspondía a colegiadas en 1935, mientras que el 39\% a matronas que no figuraban en la lista de dicho año.

Hubo 143 matronas que no estaban colegiadas en 1935, pero que solicitaron la depuración que llevaba a cabo el Colegio de Médicos de Madrid nada más acabar la guerra. Esto es debido a que algunas de ellas completaron sus estudios con posterioridad a la confección de la lista, otras recibieron la titulación de matrona durante la Guerra Civil, o tras la terminación de

\footnotetext{
66. CDMH, Tribunal de Orden Público 42/02728.

67. Libro de Actas del Colegio de Médicos de Madrid, sesiones del 25 de junio 1948 y 15 de enero de 1948, respectivamente.
} 
la contienda, y un último grupo estaría formado por aquellas que habiendo terminado sus estudios con anterioridad a 1935, no ejercieran, o no lo hicieran al menos en Madrid. Los ejemplos son numerosos: Dominga Aristu Astudillo, Mercedes Fernández Vega, Salvadora Romera y de Dios y Purificación Herrero Martín solicitaron que se les expidiese el título durante la guerra, después de abonar la tercera parte de los derechos del mismo, más la totalidad de los de timbre y expedición. Tras el informe favorable del Director de la Escuela Oficial de Matronas de Madrid, se publicó en la Gaceta de la República la expedición provisional del título de cada una de ellas. Sin embargo, sus títulos, al igual que todos los expedidos entre el 18 de julio de 1936 y al 1 de abril de 1939 en zona «no liberada», fueron anulados por orden 28 de septiembre de 1939 (BOE, 2 de octubre de 1939). Tras realizar los trámites para obtener un nuevo título estas cuatro mujeres pudieron solicitar la depuración correspondiente por el Colegio de Médicos de Madrid ${ }^{68}$.

Como para trabajar en la España autárquica se exigió durante bastantes años después la depuración favorable, el Colegio de Médicos de Madrid continuó con las de matronas hasta los años cincuenta.

\section{Matronas sancionadas por el Colegio de Médicos de Madrid}

De las más de ochocientas matronas que la Comisión depuradora del Colegio de Médicos de Madrid investigó a lo largo de doce años, desde 1941 hasta 1952, fueron sancionadas las cuarenta y ocho que se recogen en el anexo adjunto. A continuación de los apellidos y nombre de cada una de ellas se señala la sanción, la fecha de la resolución de la misma y si figuraba en la relación de asociadas del Colegio de Matronas de Madrid de 1935, con expresión del numero de asociada que tenía. Las sanciones fueron,

68. Dominga Aristu Astudillo, expedición título en la Gaceta de la República del 30 de diciembre de 1936, nueva expedición el 28 de diciembre de 1939 y depurada favorablemente en sesión del Colegio del 19 de septiembre de 1941; Mercedes Fernández Vega, primera expedición el 30 de diciembre de 1936, segunda expedición el 28 de diciembre de 1939 y depuración favorable el 12 de marz de 1941; Salvadora Romera y de Dios primera expedición el 30 de diciembre de 1936, segunda expedición el 10 de febrero de 1940 y depuración favorable el 12 de marzo de 1941; Purificación Herrero Martín primera expedición el 30 de diciembre de 1936, segunda expedición el 28 de diciembre de 1939 y depuración favorable el 12 de marzo de 1941. 
como puede comprobarse, desde la inhabilitación para ejercer cargos de confianza, a la prohibición del ejercicio profesional durante cinco años en la jurisdicción del Colegio Oficial de Matronas de la provincia de Madrid.

Si observamos el listado del anexo 1, vemos que el castigo mayor fue para Soledad Ruiz Hernando, sancionada con cinco años de privación del ejercicio profesional en Madrid e inhabilitación. Éste se produjo de manera tardía, en 1945, porque como ya hemos visto estaba encarcelada, sentenciada por un delito de adhesión a la rebelión con 30 años de prisión ${ }^{69}$; tanto su condena como su sanción probablemente se debieron a su afiliación política al Partido Comunista de España ${ }^{70}$. Las siguientes sanciones en grado de dureza las recibieron Isabel Martín Espinosa, Rosa Mora Fernández, Rosa Rivas Solano, Eufemia Llorente de Domingo, Celestina López Sevilla y Lutgarda Sánchez Hermosa, sancionadas con tres años e inhabilitación. En todas ellas es posible encontrar tareas de gestión sindical, o al menos simpatía por esta labor. Y ello debió de pesar, más que ninguna otra cosa, en que fueran sancionadas. Lutgarda Sánchez y Rosa Mora habían formado parte de la Junta Directiva del Colegio Oficial de Matronas de Madrid sito en la Plaza de Bilbao n. ${ }^{\circ}$ 12, tras su incautación en julio de 1936 por la Agrupación de Matronas de Madrid y Limítrofes, como presidenta y tesorera respectivamente ${ }^{71}$; Celestina López y Sevilla era la presidenta de dicha Agrupación de Matronas ${ }^{72}$; Eufemia Llorente de Domingo era miembro de la Agrupación de Matronas de Madrid y administradora de su órgano de expresión ${ }^{73}$; la ya citada Lutgarda Sánchez Hermosa, como presidenta de la Junta Directiva del Colegio de Matronas (comité), había recibido, en el mes de septiembre de 1936, un hotel incautado por miembros del Ateneo Libertario de la Guindalera al Marqués de Casa Real y situado en la Colonia del Retiro, en la calle de Arias Montano n. 5 y 7, con el objeto de instalar en el mismo una Maternidad de barrio $^{74}$; etcétera.

\footnotetext{
69. AGA, Justicia, Serie Tratamiento Penitenciario 7,9_01219R.jpg.

70. Hernández Sánchez, Fernando. El PCE en la Guerra Civil. Tesis doctoral. Universidad Nacional de Educación a Distancia; 2010.

71. Incautación del Colegio de Matronas de Madrid. ABC. 31 Jul 1936: 30. La Junta de dicha Agrupación, la completaba: Dolores Márquez (vicepresidenta), Pilar Pérez Ramos (secretaria general), Carmen Morales (secretaria de actas), Marina Carrión (contadora) y las vocales Remedios Rincón, Purificación de Lafuente, Adelina Bullejos, África Biencinto y Felicidad Sáez.

72. $\mathrm{CDMH}$, PS-MADRID, 473, 87.

73. Matronas. Revista técnica profesional UGT. 1933; 1 (1): 1.

74. CDMH, PS-MADRID, 473, 87.
} 
La vida de estas matronas sancionadas siguió derroteros profesionales distintos a partir de ese momento. Así, a Isabel Martín Espinosa la encontramos ejerciendo en Barcelona en $1944^{75}$ y ello, a pesar de que con fecha 15 de marzo de 1944 la Junta del Colegio acordó, a petición de la propia interesada, computarle como tiempo de cumplimiento de sanción el período que iba del 21 de febrero de 1940 al 22 de junio de 1942 en que se le notificó la resolución ${ }^{76}$. Contra Rosa Rivas Solano se siguió procedimiento sumarísimo de urgencia en la Primera Región Militar en 1942, si bien el Auditor de Guerra, al no aparecer debidamente justificada la perpetración de delito, resolvió el sobreseimiento provisional de la causa ${ }^{77}$.

En otros casos de matronas que merecieron menor sanción, como Remedios Rincón, secretaria de la Sección de Matronas del Sindicato de Sanidad e Higiene (CNT-AIT), y Ventura Marcos, presidenta de la Agrupación de Matronas UGT, también encontramos implicación sindical. Ambas, en representación de las organizaciones sindicales de matronas de la UGT y CNT, se habían dirigido por escrito al Presidente del Consejo Provincial de Madrid, el 7 de junio de $1938^{78}$, solicitando que se excluyera del programa del concurso-oposición de enfermeras los temas referentes a la especialidad profesional de matronas, en concreto los temas 26,27 y $28^{79}$.

\section{Conclusiones}

En el presente trabajo, hemos revisado el proceso de depuración política a que se vio sometido el colectivo profesional de matronas que ejercían en Madrid, realizado por diferentes instancias y que incluyó a más de ochocientas entre los años 1939 y 1952.

Las matronas que accedieron al cuerpo de funcionarias de prisiones en los años de la Segunda República, fueron las más sancionadas en todos los casos. Purificación de la Aldea y Ruiz de Castañeda y Áurea Rubio Villanueva

\footnotetext{
75. La Vanguardia. 22 Mar 1944:11. [Anuncio profesional].

76. Libro de Actas del Colegio de Médicos de Madrid, f. 79 y 79v. [15 Mar 1944].

77. CDMH, Responsabilidades Políticas, 75/01094.

78. Archivo Regional de la Comunidad de Madrid, Diputación, 149-50.

79. El enunciado del tema 26 era: Aborto. Precauciones para evitarlo y cuidados que deben presentarse por la enfermera. El del 27: Cuidados especiales para el recién nacido. Asfixia del recién nacido. Eclampsia infantil. Y el del 28: Lactancia, sus variedades. Modo de dirigirla y practicarla.
} 
fueron separadas del servicio. Catalina Mayoral Arroyo fue dada de baja en el escalafón del cuerpo de prisiones por encontrarse en paradero ignorado, si bien se encontraba encarcelada en la prisión de Ventas, la misma en la que había trabajado como funcionaria. Los motivos debemos buscarlos en que, por un lado, accedieron a su puesto en la época de la República, y, por otro, en la naturaleza represora de su trabajo, en cuyo ejercicio, durante los años que duró la contienda, interactuaron con presas políticas del bando ganador tras la Guerra Civil.

De las depuraciones instruidas por Enríquez de Salamanca sobre el personal docente de la Universidad de Madrid, fueron separadas del servicio las matronas M. ${ }^{a}$ de los Dolores Márquez Méndez y María Teresa Arroyo Nieto, esta última exiliada a México. También la pertenencia a organizaciones políticas o sindicales, así como su simpatía por el gobierno de la República, la encontramos en las matronas que merecieron mayor sanción por el Colegio de Médicos de Madrid. Soledad Ruiz Hernando, sancionada con cinco años de privación del ejercicio profesional e inhabilitación; e Isabel Martín Espinosa, Rosa Mora Fernández, Rosa Rivas Solano, Eufemia Llorente de Domingo, Celestina López Sevilla y Lutgarda Sánchez Hermosa, sancionadas con tres años e inhabilitación.

El hecho de que no fuesen los Colegios Oficiales de Matronas los encargados de depurar a sus asociadas, sino que esta labor fuese encomendada a los Colegios de Médicos de las respectivas provincias, nos muestra una diferencia clara de género con respecto al proceso depurador de otros colectivos, como es el caso de los propios médicos.

Finalmente, señalar que sería lógico pensar que entre las matronas colegiadas en 1935 que no solicitaron la depuración tras la Guerra Civil, habría muchas que se habrían significado con el gobierno de la República y que, por ello, no solicitaron la depuración ante el temor a ser represaliadas. De ellas nos ocuparemos en un próximo trabajo. 


\section{ANEXO 1}

\section{MATRONAS SANCIONADAS POR EL COLEGIO DE MÉDICOS DE MADRID}

Con expresión de la sanción, la fecha de la misma y, si figuraba en la relación del Colegio de Matronas (CM) de Madrid en 1935, con el número de asociada

Adrián Romero, Emilia: 6 meses e inhabilitación (22/06/1942) CM n. ${ }^{\circ} 93$

Álvarez Fernández, Isabel: Un año e inhabilitación (22/06/1942) CM n. ${ }^{\circ} 188$

Álvarez Martínez, Antonia: Inhabilitación (27/07/1942) CM n. ${ }^{\circ} 283$

Amador García, Sofía: 6 meses e inhabilitación (22/06/1942) CM n. 157

Arto Ortiz, Camelia: Inhabilitación (22/06/1942) CM n. ${ }^{\circ} 332$

Astiarraga Villamandos, Mercedes: Un año e inhabilitación (22/06/1942) CM n. ${ }^{\circ} 278$

Benito Román, Matilde: 6 meses e inhabilitación (22/06/1942) CM n. ${ }^{\circ} 135$

Cantudo García, Teresa: Un año e inhabilitación (22/06/1942) no CM

Domínguez Villalba, Ana: 6 meses e inhabilitación (22/06/1942) CM n. 253

Dorado Llamera, Elisa: 6 meses e inhabilitación (22/06/1942) CM n. ${ }^{\circ} 463$.

Dorado Llamera, Manuela: 6 meses e inhabilitación (22/06/1942) no CM

Fernández Muñoz, Mercedes: 6 meses e inhabilitación (22/06/1942) CM n. 397

García Escudero, María: Inhabilitación (30/11/1945) si CM n. ${ }^{\circ} 275$

González López, Isabel: 6 meses e inhabilitación (22/06/1942) CM n. ${ }^{\circ} 371$

Iglesias Rojo, Soledad: 6 meses e inhabilitación (22/06/1942) CM n. ${ }^{\circ} 294$

Jerónimo Sanz, Antonia: Inhabilitación (22/06/1942) CM n. 378

Lencina Muñoz, Josefa: 6 meses e inhabilitación (22/06/1942) no CM

Liñán Lozano, Magdalena: Inhabilitación (20/06/1944) CM n. ${ }^{\circ} 441$

Llorente de Domingo, Eufemia: Tres años e inhabilitación (22/06/1942) CM n. ${ }^{\circ} 161$

López Díaz, Agapita: Inhabilitación (22/06/1942) no CM

López Sevilla, Celestina: Tres años e inhabilitación (22/06/1942) CM n. ${ }^{\circ} 132$

Maeztu Hita, Eulalia: Inhabilitación (22/06/1942) CM n. ${ }^{\circ} 179$

Marcos Villarreal, Ventura: Un año e inhabilitación (22/06/1942) CM n. ${ }^{\circ} 462$

Mari Morante, Pilar: Inhabilitación (22/06/1942) no CM

Martín Espinosa, Isabel: Tres años e inhabilitación (22/06/1942) CM n. ${ }^{\circ} 66$

Martín García, Juana: 6 meses e inhabilitación (22/06/1942) CM n. ${ }^{\circ} 344$

Martínez de la Rosa, Mariana: Inhabilitación (22/06/1942) CM n. ${ }^{\circ} 6$

Melgares Mateo, Encarnación Dos años e inhabilitación (22/06/1942) CM n. ${ }^{\circ} 60$

Mora Fernández, Rosa: Tres años e inhabilitación (22/06/1942) CM n. ${ }^{\circ} 36$

Moreda Isarre, Florentina: Inhabilitación (22/06/1942) CM n. ${ }^{\circ} 274$

Muñoz González, Concepción: Inhabilitación (22/06/1942) CM n. ${ }^{\circ} 83$

Palomino Cid, Carmen: Inhabilitación (17/12/1945) CM n. ${ }^{\circ} 98$

[Con posterioridad fue depurada favorablemente (15/12/1946)]

Pérez Ramos, Pilar: 6 meses e inhabilitación (22/06/1942) CM n. ${ }^{\circ} 159$ 
Polán Cordero, Carmen: 6 meses e inhabilitación (22/06/1942) no CM

Portela Carrillo, Ana Dolores: Un año e inhabilitación (22/06/1942) CM n. 138

Ramírez, María Ana: 6 meses e inhabilitación (22/06/1942) no CM

Ramírez Blanco, Luisa: 6 meses e inhabilitación (22/06/1942) CM n. ${ }^{\circ} 479$

Ramírez Fernández, Polonia Rosario: Inhabilitación (17/12/1945) CM n. ${ }^{\circ} 426$

Rey López, María: Inhabilitación (22/06/1942) CM n. 478

Rincón Fernández, Remedios: Inhabilitación (22/06/1942) CM n. ${ }^{\circ} 124$

Rivas Solano, Rosa: Tres años e inhabilitación (22/06/1942) CM n. ${ }^{\circ} 148$

Ruiz Hernando, Soledad: Cinco años e inhabilitación (9/01/1945) CM n. 343

Sáez Hernández, Felicidad: Inhabilitación (15/01/1948) no CM

San Julián Sánchez, Celestina: Inhabilitación (22/06/1942) no CM

Salamanca Escudero, Aurelia: 6 meses e inhabilitación (22/06/1942) CM n. ${ }^{\circ} 58$

Sánchez Hermosa, Lutgarda: Tres años e inhabilitación (22/06/1942) CM n. ${ }^{\circ} 117$

Segura de Medina, Enriqueta: Inhabilitación (22/06/1942) CM n. 267

Valero Cano, Milagros: Inhabilitación (22/06/1942) CM n. ${ }^{\circ} 463$

Fuente: Libros de actas del Colegio Oficial de Médicos de Madrid, desde el 17 de octubre de 1940 al 16 de agosto de 1952. Elaboración propia. 
1.- Introduction. 2. -The implication of several departments in the purge of the midwives of Madrid. 3.-Purge and sanction of the workers in the Central University.4.-Purge and sanction of the midwives who acted as prison officers. 5.-Purge of the midwives by the College of Physicians of Madrid. 6.- Midwives punished by the College of Physicians of Madrid. 7.-Conclusions.

ABSTRACT: Midwives in Madrid became incorporated in different positions in the Administration during the years before the Civil War, explaining why the post-war purge took place in various branches. This study highlights the purge of midwives working in different departments of the Central University, those working as prison functionaries or similar occupations, and those purged by the College of Physicians of Madrid, who formed the majority. After explaining how the purge dossiers were compiled by the corresponding examining magistrate and describing the results obtained in each case, we name the midwives who received the most severe punishments and discuss the reasons given in each case. 